\title{
THE CONTRAST GAIN CONTROL OF THE CAT RETINA
}

\author{
ROBERT SHAPLeY and Jonathan D. Victor \\ The Rockefeller University, New York, NY 10021, U.S.A.
}

This paper is about a phenomenon, and a mechanism to explain the phenomenon. The observation is that the dynamic transfer properties of the cat retina are altered when the contrast of the visual stimulus is changed. The peculiar dependence of the "contrast effect" on the spatial and temporal properties of the visual stimulus led us to the conclusion that the contrast effect is caused by a separate and distinct retinal mechanism. We call this mechanism the contrast gain control.

Our experiments are performed on single fibers in the optic tract of cats anesthetized with urethane and paralyzed with gallamine and alloferin (cf. Hochstein and Shapley, 1976a for details). The visual stimulus is generated on a cathode ray tube (CRT) at a mean luminance of $20 \mathrm{~cd} / \mathrm{m}^{2}$. We classify the optic tract fibers as $\mathrm{X}$ or $\mathrm{Y}$ on the basis of their responses to contrast reversal of fine sinusoidal gratings. As spatial frequency of the grating is increased, the Y-cell response becomes predominantly nonlinear. The symptoms of this nonlinearity are that the cell's response is mainly at twice the modulation frequency and is completely independent of the position, or spatial phase, of the grating stimulus (Enroth-Cugell and Robson, 1966; Hochstein and Shapley, 1976a). On the other hand, $\mathrm{X}$-cells behave in a linear manner. They respond at the modulation frequency, and this fundamental response is a sinusoidal function of spatial phase. For classification, the modulation signal which controls the instantaneous value of the contrast of the grating pattern is a single sine-wave around $4 \mathrm{~Hz}$.

After the unit is classified, we change the temporal modulation signal to a sum of eight sinusoids of equal amplitude. These sinusoids are roughly a factor of 2 apart in frequency and span the range $0.2-31 \mathrm{~Hz}$ (cf. Victor, Shapley and Knight, 1977). When the experimenter looks at the CRT screen at a sine grating modulated by this sum of sinusoids, the grating appears to be varying in contrast in a random manner. If one listens to the response of an optic tract fiber to this visual stimulus, one hears its firing rate modulated in what seems a random manner. But the appearance of randomness is an illusion.

One can recover the linear, or first order, response of the ganglion cell by Fourier analysis. One looks for Fourier components at the input temporal frequencies in the sum of sinusoids. One can also recover the best estimate of the nonlinear second order responses of the cell by looking at the Fourier components at sums and differences of the input frequencies (Victor et al., 1977). Second order responses will be discussed below. At first we wish to focus on the first order frequency response contained in the re- sponse amplitudes and phases at the input frequencies.

Two first order frequency responses from a cat $Y$ ganglion cell are shown in Fig. 1. In one experimental run the depth of modulation of the sinusoids was eight times higher than the other. The depth of the modulation is equivalent to the peak contrast the grating reaches. The contrast figures for the two frequency responses in Fig. 1 are 0.0125 peak contrast per sinusoid (root mean square (rms) average contrast of 0.025 for the sum of eight sinusoids) and 0.1 per sinusoid (rms average contrast of 0.2 ). Clearly, the frequency response is affected by contrast; at higher

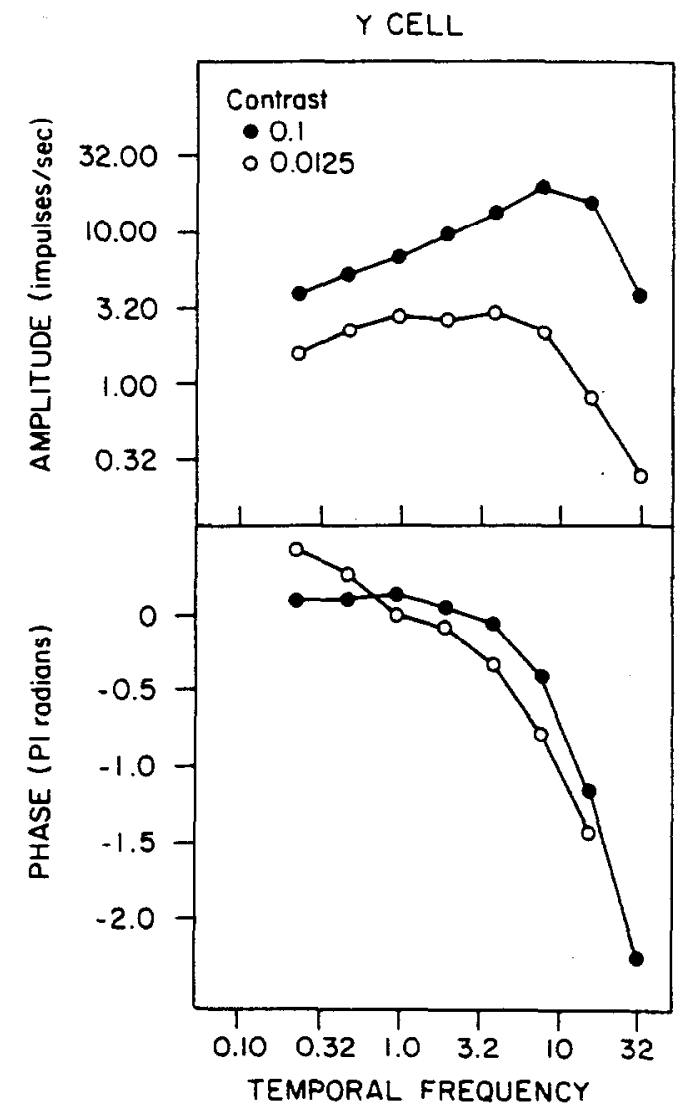

Fig. 1. The effect of contrast on the first order frequency response. These are data from an on-centre $\mathrm{Y}$ retinal ganglion cell. The mean luminance was $20 \mathrm{~cd} / \mathrm{m}^{2} ;$ the contrasts of the stimuli were $0.0125 /$ sinusoid $(0)$ or $0.1 /$ sinusoid $(\bullet)$. The temporal modulation signal was the sum of eight sinusoids. The spatial pattern was a sine grating with a spatial. frequency $0.25 \mathrm{c} / \mathrm{deg}$. 


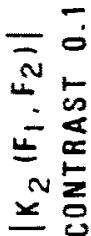
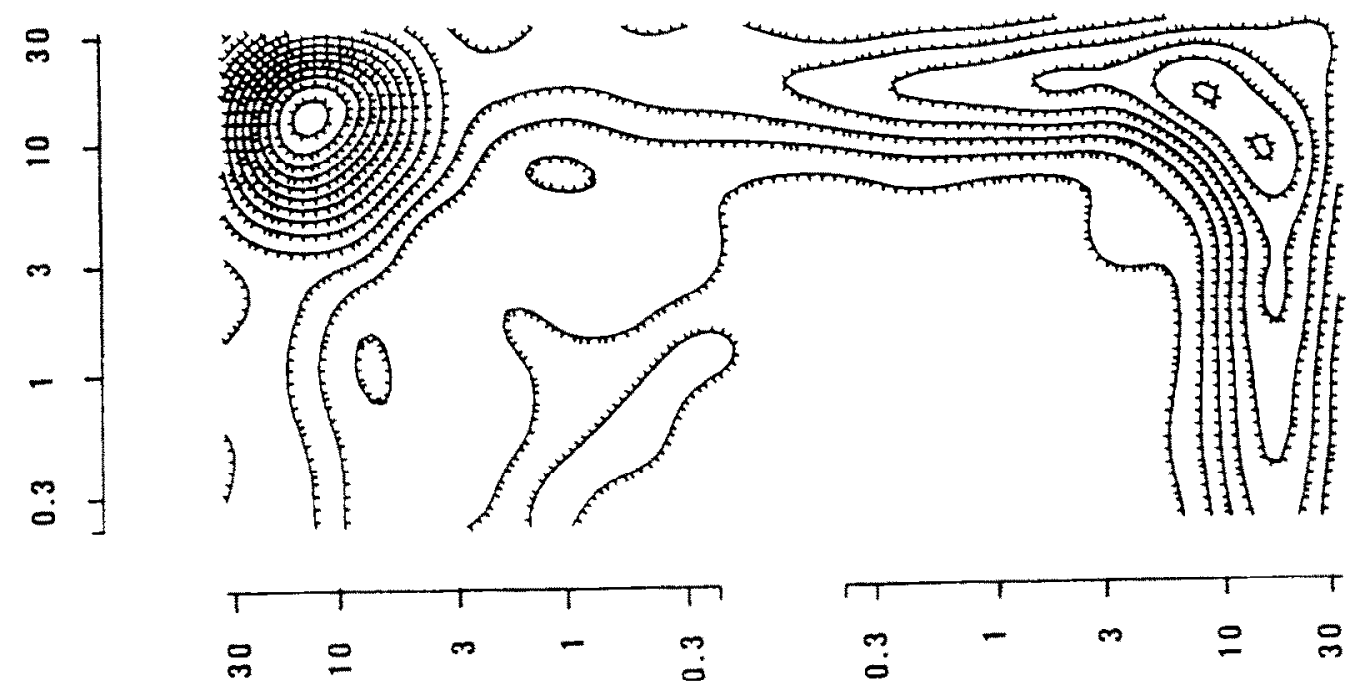

$3=2$

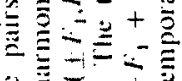

$\underline{\underline{z}}$

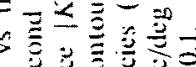

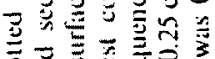

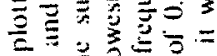

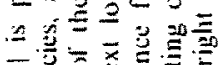

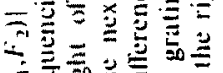

$+1$

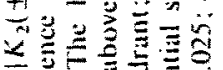

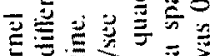

प.

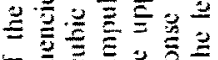

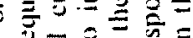

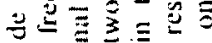

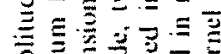

只 0 \%

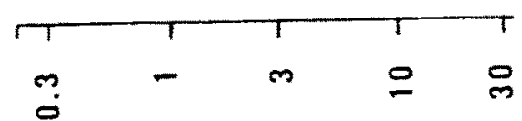

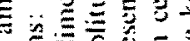

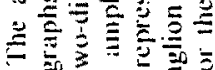

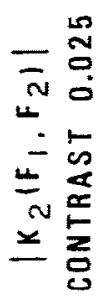

0
0
0
-
0

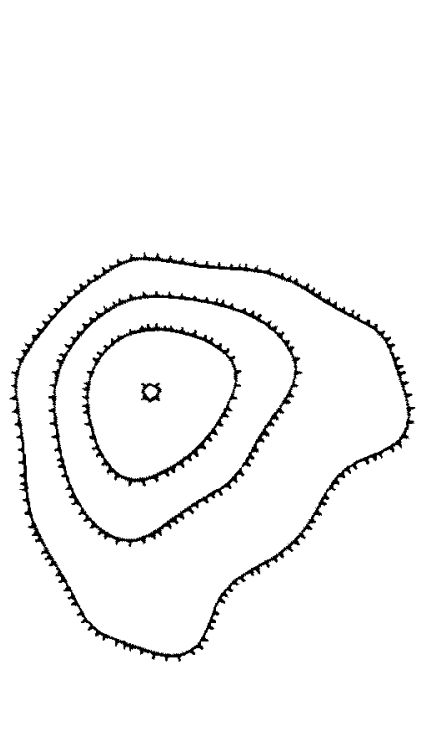

3

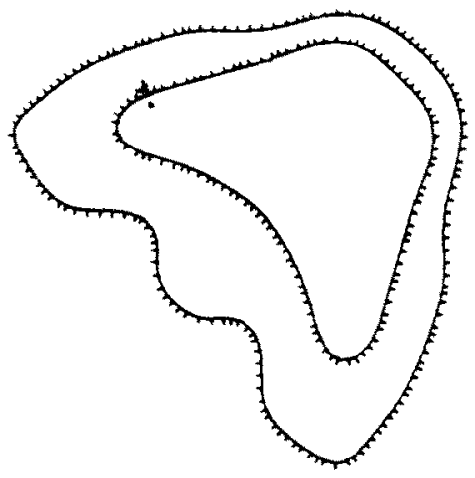

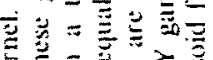

$3=0$

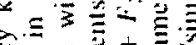

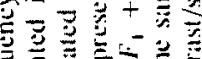

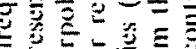

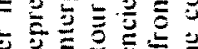

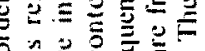

ว

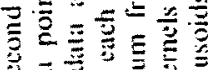

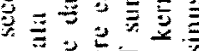

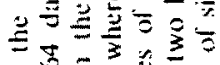

亏

$x$

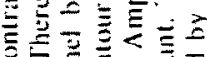

Em

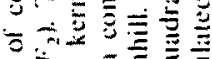

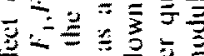

$+1 \div-5$

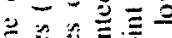

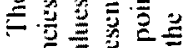

八

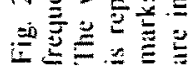

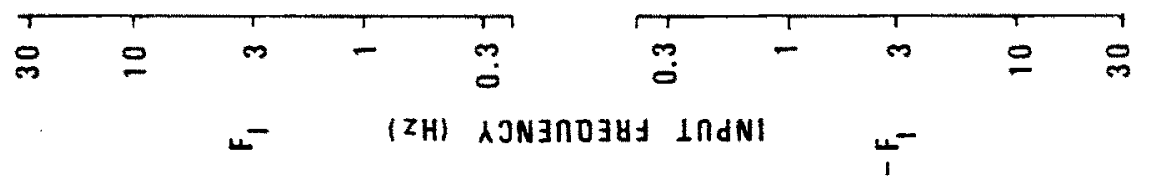




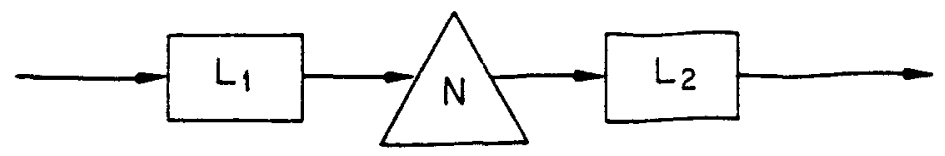

Fig. 3. A linear, nonlinear linear "sandwich" model. This is a model which may apply to the nonlinear pathway of the $Y$ retinal ganglion cells. $L_{1}$ is a linear filter, the prefilter. $N$ is a nonlinear element.

In the retina $\mathrm{N}$ seems to be a rectifier or threshold element. $L_{2}$ is a linear filter. the post-filter.

contrast, the amplitude curve is more sharply tuned and peaks at a higher temporal frequency. The reason for the amplitude curve changes is that the amplitudes of high frequency components are roughly proportional to contrast, while the amplitudes of low temporal frequency components grow less than proportionally with contrast. Furthermore, there is a consistent phase advance at high temporal frequencies. In terms of the magnitude of the phase advance the contrast effect is twice as great on Y-cells as on X-cells.

The spatial and temporal dependence of the contrast effect define the peculiar nature of the contrast gain control. The magnitude of the contrast effect is a relatively flat function of spatial frequency, usually peaking around $0.25 \mathrm{c} / \mathrm{deg}$. The total contrast averaged over time determines the contrast effect. This is what we find when we compare frequency-response curves measured with single sinusoids with those measured with eight sinusoids. The single sinusoid curves show much less change with contrast/sinusoid. 'This result implies that stimulus power at one temporal frequency can modify the amplitude and phase of the response at other temporal frequencies. One can use this fact to show that the best temporal frequencies for producing the contrast effect are between 4 and $15 \mathrm{~Hz}$ (Shapley and Victor, 1978).

One can also show that the contrast effect depends on the local retinal contrast averaged over a wide region of space, because the contrast effect does not depend on the spatial phase of the grating used as a stimulus. This peculiar spatial-phase-independence of the contrast gain control immediately suggests that it is in some way related to the neural mechanisms we have called the "nonlinear subunits", which provide the nonlinear, excitatory input to $Y$-cells (Hochstein and Shapley, 1976b). The observation of spatialphase-independence is incompatible with explanations of the contrast effect in terms of a neural mechanism with only a single spatial profile. The spatialphase-independence forces one to think in terms of many subunits. Furthermore, it rules out magnitude of the first order response as having anything to do with the contrast effect, because magnitude of the first order response in both $\mathrm{X}$ - and $\mathrm{Y}$-cells is dependent on the spatial phase of the grating stimulus (Hochstein and Shapley, 1976a; Victor et al., 1977).

In Y-cells one can also measure large second order non-linear responses as shown in Fig. 2. Each graph in Fig. 2 shows a contour map of a surface, that surface being the amplitude of the second order frequency response as a function of the set of frequency

${ }^{t}$ However, if the tms average contrast of the single sinusoids is equated to the rms average of the sum of sinusoids. then the frequency responses determined with the single sinusoids are approximately equal to the first order frequency responses measured with the sum of sinusoids. pairs $\left( \pm F_{1}, F_{2}\right)$ (cf. Victor et al.. 1977). These frequency pairs are pairs of the input frequencies which are added and subtracted to make the second order crosstalk frequencies. The contours represent nonlinear responses of constant amplitude. and the tick marks point downhill. In these graphs each contour line represents an increment of 2 impulses $/ \mathrm{sec}$. Thus, in Fig. 2A there are two hills in the second order frequency response with peaks in both the sum $\left(F_{1}, F_{2}\right)$ and difference frequency $\left(-F_{1}, F_{2}\right)$ quadrants at around $8 \mathrm{~Hz}$ input frequency. The reason for these hills can be understood in terms of the model in Fig. 3. This is a sandwich of a nonlinearity $N$ between two linear filters: a pre-filter $\mathrm{L}_{1}(f)$ and a post-filter $\mathrm{L}_{2}(f)$. One can show that if the pre-filter $\mathrm{L}_{1}(f)$ is a bandpass filter. then there must be hills in the second order frequency kernel when both frequencies in the pair $\left( \pm F_{1}, F_{2}\right)$ are near the peak of $L_{t}(f)$ (Victor et al., 1977). The post-filter $\mathrm{L}_{2}(f)$ introduces asymmetry between the heights and shapes of the hills in the sum and difference frequency quadrants. We also should add that we believe the model in Fig. 3 has some physiological significance. We believe that the prefilter in the "sandwich model" of the nonlinear excitatory pathway in the cat retina may be the bipolar cell, and the nonlinearity and post-filter may be associated with amacrine cells (Victor et al., 1977).

The second order frequency responses in Fig. 2 exhibit a contrast effect similar to that shown by first order responses. The contrasts used to elicit the two responses in Fig. 2 were separated by a factor of 4 . Notice that, in the second order frequency response at higher contrast, on the right, the peaks of the hills in the kernel have moved up. Instead of peaking at around $8 \mathrm{~Hz}$, they are both now peaking around $12 \mathrm{~Hz}$. This is the second order contrast effect. Like the first order contrast effect, it is spatial-phaseinsensitive, i.e. the spatial phase of the stimulus grating does not affect the second order frequency response. The effect of contrast on second order amplitudes is associated with substantial temporal phase advances, as in the first order contrast effect. However, there is a wonderful difference between phase advances of sum frequencies and difference frequencies which allows us to be very specific about how the contrast gain control works. The difference frequencies usually have much less phase advance than the sum frequencies-in some cases none at all. One can show that this implies that the adjustment of the dynamic transfer properties in the nonlinear excitatory pathway is taking place before the dominant nonlinear transduction which generates the sum and difference frequencies. In terms of the "sandwich model" (Fig. 3), the major point of contrast adjustment is on the filter $L_{1}$. We have already argued above that the contrast gain control acted as if it were located after the major nonlinearity in the cat 
retina. Thus, one reasonable working hypothesis is that the contrast gain control feeds back from after the nonlinearity to neural stages before the nonlinearity. We think that such a (feedback) contrast gain control mechanism may be present in many if not all vertebrate retinae (cf. Werblin and Copenhagen. 1974).

The intricacies of the mechanism of the contrast gain control have absorbed us, but now let us consider its fundamental importance in vision. Not only ambient light, but also ambient contrast serves to adjust the sensitivity of the retina. At higher contrasts the response of the cat retina becomes more "transient" in that slow variations are suppressed, and more rapid variations are enhanced. The responses of a ganglion cell to slow modulations may be suppressed, and the cell's responses to rapid changes speeded up, by contrasts many degrees away in the visual field (Shapley and Victor. 1979). Thus, visual perception must be altered in a complex manner by the arrangement of contrasts in the visual scene. Mechanisms similar to the retinal contrast gain control may be present at all levels of perception. For, as Portia says in The Merchant of Venice.

So doth the greater glory dim the less.

A substitute shines brightly as a king

Until a king be by:...

Nothing is good I see without respect...

(Shakespeare. 1596).
Acknowitedgements-This work wals supported by research grants from the National Eye Institute: EY 1472. EY 188 and EY 1428. R.S. was supported by a Career Development Award from the Nittional Eye Institute.

\section{REFERENCES}

Enroih-Cugell C. and Robson J. G. (1966) The contrast sensitivity of retinal ganglion cells of the cat. J. Phrsiol. 187. $517-552$.

Hochstein S. and Shapley R. M. (1976a) Quantitative analysis of retinal ganglion cell classifications. J. Physiol. 262, 237-264.

Hochstein S. and Shapley R. M. (1976b) Linear and nonlinear spatial subunits in $Y$ cat retinal ganglion cells. J. Physiol. 262, 265-284.

Shakespeare W. (1596) The Merchant of Venice. Act V, Scene 1. 1.93-99.

Shapley R. M. and Victor J. D. (1978) The effect of contrast on the transfer properties of cat retinal ganglion cells. J. Physiol. In press.

Shapley R. M. and Victor J. D. (1979) Nonlinear spatial summation and the contrast gain control in cat retinal ganglion cells, $J$. Phisiol. In press.

Victor J. D., Shapley R. M. and Knight B. W. (1977) Nonlinear analysis of cat retinal ganglion cells in the frequency domain. Proce natn. tcad. Sci. L.S.t.. 74. 3068-3072.

Werblin F. and Copenhagen D. (1974) Control of retinal sensitivity. III. Lateral interactions at the inner plexiform layer $J$ gen. Physiol. 63, 88-110. 